involving GI and CNS. Therapy involves plasma exchange to replace complement factors and more recently the use of C5 inhibitors that have been highly effective.

In those who progress to end-stage renal failure, transplantation carries a high risk of disease recurrence and this is highest with CFH mutations.

Exclusion of infectious HUS and of TTP, a similar adult disease due to defects in ADAMTS13, is important. Genetic diagnosis has been significantly aided by massively parallel sequencing that can sequence the 10 known genes in one run. Further analysis using MLPA to identify rearrangements and deletions is also increasingly of use. Genetic identification assists in risk stratification and in identifying those children who will benefit from C5 inhibitors.

\section{MAKING A MEAL OF MULTIPLE MUTATIONS (AND OTHER MORPHOLOGIC AND MOLECULAR MALADIES) IN MYELOID MALIGNANCIES I: ACUTE MYELOID LEUKEMIA}

\author{
Adam Bagg \\ Department of Pathology and Laboratory Medicine, University \\ of Pennsylvania, Philadelphia, PA, USA
}

The laboratory evaluation of acute myeloid leukaemia (AML) is multiparametric and includes a number of different technological approaches including morphology, cytochemistry (sometimes), immunophenotyping and genetic studies. Amongst these tools, it has become quite clear that genetic evaluation carries the greatest relevance with regard to prognosis and hence therapy. It is thus not surprising that contemporary classification incorporates genetic data into the diagnosis of some forms of AML. However, the genetic abnormalities are diverse, and AML is an extremely heterogeneous disease at the genetic level, with, for example, at least 160 different but recurrent structural cytogenetic abnormalities observed, and dozens of genes that may be targeted for mutation. Dysregulation may also occur at the epigenetic and microRNA level. Many of the genetic underpinnings of AML can be routinely dissected in the clinical laboratory via a variety of different but often complementary strategies ranging from karyotypic metaphase analysis, polymerase chain reaction-based approaches to targeted massively parallel sequencing approaches. This lecture will review the added value afforded by genetic studies in the assessment of AML, while at the same time not losing sight of the value of morphology.

\section{MAKING A MEAL OF MULTIPLE MUTATIONS (AND OTHER MORPHOLOGIC AND MOLECULAR MALADIES) IN MYELOID MALIGNANCIES II: MYELOPROLIFERATIVE NEOPLASMS}

\section{$\underline{\text { Adam Bagg }}$}

Department of Pathology and Laboratory Medicine, University of Pennsylvania, Philadelphia, PA, USA

The classical myeloproliferative neoplasms (MPNs) consist of chronic myelogenous leukaemia (CML) and the non-CML
MPNs, polycythaemia vera (PV), essential thrombocythaemia (ET) and primary myelofibrosis (PMF), while the so-called nonclassical subtypes include chronic neutrophilic leukaemia and various flavours of mast cell neoplasms. Another group incorporates those associated with eosinophilia and recurrent genetic abnormalities. A fourth category encompasses MPNs that show features that overlap with the myelodysplastic syndromes, such as chronic myelomonocytic leukaemia, juvenile myelomonocytic leukaemia and atypical chronic myeloid leukaemia. Molecular testing plays a role in each of these entities. This lecture will focus on the value of the various modalities for detecting the hallmark genetic abnormality of CML, the $\mathrm{t}(9 ; 22) / B C R-A B L 1$, at diagnosis and monitoring, as well as testing for $A B L 1$ mutations in the setting of resistance to tyrosine kinase inhibitor therapy. Many of the genetic mysteries of the non-CML MPNs have been unravelled in the past decade, and the role of $J A K 2, M P L$ and $C A L R$ mutations in PV, ET and PMF will also be reviewed. The discovery of recurrent (though not always diagnostically-specific) genetic lesions in some of the other neoplasms that may fall under the broad umbrella of MPNs facilitates their diagnosis, prognosis and ultimately therapy.

\section{PROGRESS IN GENE THERAPY FOR GENETIC DISEASES}

John Rasko $\mathrm{AO}^{1,2}$

${ }^{I}$ Cell and Molecular Therapies, Royal Prince Alfred Hospital, Camperdown, and ${ }^{2}$ Gene and Stem Cell Therapy Program Centenary Institute, Sydney Medical School, University of Sydney, NSW, Australia

It is an exciting time for genetic and cellular therapies. Since 1989 over 1500 Phase I/II studies of direct in vivo and cellmediated gene therapy in diverse diseases have been completed, with a few entering the market. Substantial evidence of improved clinical outcomes has been shown in haemophilia B, immune deficiencies, haemoglobinopathies, immunotherapies and blindness. Our experience in AAV-mediated gene therapy for haemophilia has led to a recent clinical focus on beta thalassaemia major. Transplantation of patients with severe thalassaemia using autologous $\mathrm{CD}^{+} 4^{+}$cells transduced with LentiGlobin vector has provided preliminary evidence of safety and efficacy. Indeed, if gene-mediated induced pluripotent cells can be differentiated ex vivo to recreate and repair mature human tissues then regenerative medicine will become a reality. ${ }^{1}$ With gene and stem cell tourism becoming a multi-billion dollar industry, the administration of unregulated, undefined, untested or unsafe 'therapeutics' places the field at a challenging crossroad. ${ }^{2}$ References

1. Hussein SM, Puri MC, Tonge PD, et al. Genome-wide characterization of the routes to pluripotency. Nature 2014; 516: 198-206.

2. Dominici M, Nichols K, Srivastava A, et al. Positioning a scientific community on unproven cellular therapies: the 2015 International Society for Cellular Therapy perspective. Cytotherapy 2015; 17: 1663-6. 